Louisiana State University

LSU Digital Commons

Faculty Publications

Department of Mathematics

5-7-2009

\title{
Uniform global asymptotic stability of a class of adaptively controlled nonlinear systems
}

\author{
Frédéric Mazenc \\ INRAE's Occitanie-Montpellier Centre \\ Marcio de Queiroz \\ Louisiana State University \\ Michael Malisoff \\ Louisiana State University
}

Follow this and additional works at: https://digitalcommons.Isu.edu/mathematics_pubs

\section{Recommended Citation}

Mazenc, F., de Queiroz, M., \& Malisoff, M. (2009). Uniform global asymptotic stability of a class of adaptively controlled nonlinear systems. IEEE Transactions on Automatic Control, 54 (5), 1152-1158. https://doi.org/10.1109/TAC.2009.2013053

This Article is brought to you for free and open access by the Department of Mathematics at LSU Digital Commons. It has been accepted for inclusion in Faculty Publications by an authorized administrator of LSU Digital Commons. For more information, please contact ir@lsu.edu. 


\title{
Uniform Global Asymptotic Stability of a Class of Adaptively Controlled Nonlinear Systems
}

\author{
Frédéric Mazenc Marcio de Queiroz Michael Malisoff
}

\begin{abstract}
We give a new explicit, global, strict Lyapunov function construction for the error dynamics for adaptive tracking control problems, under an appropriate persistency of excitation condition. We then allow time-varying uncertainty in the unknown parameters. In this case, we construct input-to-state stable Lyapunov functions under suitable bounds on the uncertainty, provided the regressor also satisfies an affine growth condition. This lets us quantify the effects of uncertainties on both the tracking and the parameter estimation. We illustrate our results using Rössler systems.
\end{abstract}

\section{INTRODUCTION}

Consider a nonlinear system

$$
\dot{x}=f(t, x, \theta, u)
$$

where $\theta$ is a vector of uncertain constant parameters. The adaptive tracking control problem for (1) is: Given a sufficiently smooth reference trajectory $x_{r}(t)$, find a dynamic feedback

$$
u=u(t, x, \hat{\theta}), \quad \dot{\hat{\theta}}=\tau(t, x, \hat{\theta}),
$$

where $\hat{\theta}$ is the estimate of $\theta$, that ensures that $x_{r}(t)-x(t) \rightarrow 0$ as $t \rightarrow \infty$ while keeping all closed-loop signals bounded. In general, solving the adaptive tracking problem does not guarantee that $\theta-\hat{\theta}(t) \rightarrow 0$ as $t \rightarrow \infty$; i.e., parameter identification is not assured. In fact, one does not know in general whether $\hat{\theta}$ even converges to a constant vector [5].

Mazenc is with Projet MERE INRIA-INRA, UMR Analyse des Systèmes et Biométrie INRA, 2 pl. Viala, 34060 Montpellier, France, Frederic.Mazenc@supagro.inra.fr. de Queiroz is with the Department of Mechanical Engineering, Louisiana State University, Baton Rouge, LA 70803-6413, dequeiroz@me.lsu.edu. Malisoff is with the Department of Mathematics, Louisiana State University, Baton Rouge, LA 70803-4918, malisoff@1su.edu. Supported by NSF/CAREER Grant 0447576 (MdQ) and NSF/DMS Grants 0424011 and $0708084(\mathrm{MM})$. 
Persistency of excitation (PE) has been linked to the asymptotic stability of adaptive systems [13]. PE establishes that a necessary (and sometimes sufficient) condition for parameter identification is that the reference trajectory be sufficiently rich so that the regressor satisfies a PE inequality [3] along the reference trajectory. For large classes of systems, PE implies that tracking error convergence can only happen when the adaptation law identifies the actual parameters [15]. The relation between parameter identification, uniform asymptotic stability, and PE was first shown for linear systems, and has been established for certain types of nonlinear systems. (Uniformity with respect to initial times has important implications for robustness. For example, this property ensures stability in the face of persistent disturbances [2] and provides rate of convergence information [12]. In general, PE is neither necessary nor sufficient for uniform asymptotic stability [13].) One notable example is the nonlinear dynamics of robot manipulators, where PE ensures asymptotic parameter error convergence under the Slotine-Li adaptive controller [15]. Recently, PE was shown to be necessary and sufficient for uniform global asymptotic stability (UGAS) of a class of nonlinear systems that includes the manipulator dynamics [6], [7].

When an adaptive controller does not yield GAS, this means that the corresponding closedloop system does not admit a strict Lyapunov function (as defined precisely in the next section). However, even when the controller yields UGAS, the classical Lyapunov approach does not give an explicit strict Lyapunov function. Explicit strict Lyapunov functions are generally more useful than nonstrict ones when computing stability gains or quantifying the effects of uncertainty.

The present work provides a global, explicit, strict Lyapunov function for the error dynamics for adaptive tracking problems under a PE condition. It belongs to a family of results that transform nonstrict Lyapunov functions into explicit strict Lyapunov functions; see [9], [10] for constructions of this type for large classes of time-invariant systems. The paper [11] contains a very general result on constructing strict Lyapunov functions for nonlinear time-varying systems for which so-called auxiliary functions are known; i.e., the strict Lyapunov function construction in [11] is nonexplicit, unless the auxiliary functions are known.

By contrast, the present work provides explicit expressions for auxiliary functions, which make our Lyapunov function completely explicit. The Lyapunov functions we obtain here are much simpler than the ones that would be obtained by applying [11]. Finally, the Lyapunov functions we provide here are lower bounded near 0 by positive definite quadratic functions, while the Lyapunov function construction of [11] would not have this property. We also use the idea of weighting functions, which have been used in other contexts [1], [4], [19]. The global strict 
Lyapunov-based framework can potentially generalize the UGAS proofs for adaptive systems. This paper takes the first step towards this generalization.

\section{Definitions, Standing Assumptions, And Notation}

For a given vector $\theta \in \mathbb{R}^{p}$ of unknown constant parameters, we consider dynamical systems

$$
\dot{x}=\omega(x) \theta+u \quad\left(x, u \in \mathbb{R}^{n}\right) .
$$

Fix a $C^{1}$ (=continuously differentiable) function $x_{r}: \mathbb{R} \rightarrow \mathbb{R}^{n}$ which we call a reference trajectory. Let $|\cdot|_{\infty}$ denote the essential supremum, $I_{p}$ denote the $p \times p$ identity matrix, and $\mathbb{R}^{n \times p}$ denote the set of all $n \times p$ real matrices. For square matrices $M$ and $N$ of the same size, $M \geq N$ means $M-N$ is nonnegative definite. We make the following two assumptions throughout:

Assumption 1: There is a known constant $B>0$ such that $\max \left\{\left|x_{r}\right|_{\infty},\left|\dot{x}_{r}\right|_{\infty}\right\} \leq B$.

Assumption 2: The entries $\omega_{i j}$ of $\omega=\left[\omega_{i j}\right]: \mathbb{R}^{n} \rightarrow \mathbb{R}^{n \times p}$ are $C^{1}$. Also, there are known positive constants $\mu$ and $T$ such that $\mu I_{p} \leq \int_{t-T}^{t} \omega\left(x_{r}(l)\right)^{\top} \omega\left(x_{r}(l)\right) \mathrm{d} l$ for all $t \in \mathbb{R}$.

Assumption 2 is the classical PE condition [6]. We use the functions $\bar{\omega}(l)=\max \{|\omega(z)|:|z| \leq$ $l\}$ and $\bar{\omega}_{1}(l)=\sup \left\{\left|\frac{d}{d t} \omega(\sigma(t))\right|_{\infty}: \sigma \in C^{1}, \max \left\{|\sigma|_{\infty},|\dot{\sigma}|_{\infty}\right\} \leq l\right\}$, where $|\cdot|$ is the Euclidean norm or induced matrix norm, depending on the context. Then $\left|\frac{d}{d t} \omega\left(x_{r}\right)\right|_{\infty} \leq \bar{\omega}_{1}(B)$.

A real valued function $r \mapsto \alpha(r)$ is positive semi-definite provided (i) $\alpha(s) \geq 0$ for all $s$ and (ii) $\alpha(0)=0$. If, in addition, $\alpha(s)=0$ if and only if $s=0$, then we say that $\alpha$ is positive definite. If $\alpha(s) \rightarrow+\infty$ as $|s| \rightarrow+\infty$, then we say that $\alpha$ is proper. A function $\alpha$ is called negative definite provided $-\alpha$ is positive definite; negative semi-definiteness is defined analogously. A function $\alpha(t, z)$ is uniformly proper and positive definite provided $z \mapsto \inf _{t} \alpha(t, z)$ is proper and positive definite. We use the standard classes of comparison functions $\mathcal{K}_{\infty}$ and $\mathcal{K} \mathcal{L}$; see [18].

By a nonstrict Lyapunov function for a system $\dot{z}=\mathcal{G}(z, t)$ evolving on $\mathbb{R}^{n}$, we mean a $C^{1}$ uniformly proper and positive definite function $V(t, z)$ that admits a positive semi-definite function $W(z)$ such that $\frac{d}{d t} V(t, z) \leq-W(z)$ along all of its trajectories. If, in addition, $W$ is positive definite, then $V$ is a strict Lyapunov function for the system. (We always assume that $z(t)$ is uniquely defined on $\left[t_{o}, \infty\right)$ for all initial conditions $z\left(t_{o}\right)=z_{o}$, and that our systems are all sufficiently smooth.) The system is locally exponentially stable to 0 provided there exist positive constants $\underline{c}$ and $\bar{c}$ such that $|z(t)| \leq \bar{c} e^{-\underline{c}\left(t-t_{o}\right)}\left|z\left(t_{o}\right)\right|$ for all $t \geq t_{o} \geq 0$ and all trajectories $z(t)$ of the system with initial values $z\left(t_{o}\right) \in \underline{c} \mathcal{B}_{n}\left(=\right.$ closed ball in $\mathbb{R}^{n}$ of radius $\underline{c}$ centered at 0$)$.

Let $\bar{\delta}>0$ be a given constant. By input-to-state stability (ISS) [17] of a system $\dot{z}=f(t, z, \delta)$ with respect to a measurable essentially bounded uncertainty $\delta: \mathbb{R} \rightarrow \bar{\delta} \mathcal{B}_{p}$, we mean that there 
are $\beta \in \mathcal{K} \mathcal{L}$ and $\gamma \in \mathcal{K}_{\infty}$ such that the ISS estimate $|z(t)| \leq \beta\left(\left|z\left(t_{o}\right)\right|, t-t_{0}\right)+\gamma\left(|\delta|_{\infty}\right)$ holds for all $t \geq t_{o}$, trajectories $z(t)$ of $\dot{z}=f(t, z, \delta)$, initial times $t_{0} \geq 0$, and $\delta$ 's. We assume that $z(t)$ is uniquely defined on $\left[t_{o}, \infty\right)$ for all $\delta$ and all initial conditions $z\left(t_{o}\right)=z_{o}$, and that $f(t, 0,0) \equiv$ 0 . When $\delta(t) \equiv 0$, the ISS condition reduces to uniform global asymptotic stability (UGAS). Following convention, we also use ISS to mean input-to-state stable, and we also use UGAS to mean uniformly globally asymptotically stable. An ISS Lyapunov function for $\dot{z}=f(t, z, \delta)$ is a $C^{1}$ uniformly proper and positive definite function $V(t, z)$ that admits a proper positive definite function $W(z)$ and a function $\alpha \in \mathcal{K}_{\infty}$ such that $\frac{d}{d t} V(t, z) \leq-W(z)+\alpha(|\delta(t)|)$ along all trajectories of $\dot{z}=f(t, z, \delta)$ for all uncertainties $\delta(t)$. A function $\mathcal{F}(t, d, p)$ is uniformly bounded in $p$ provided there is a positive increasing function $\alpha$ such that $|\mathcal{F}(t, d, p)| \leq \alpha(|p|)$. Here and in the sequel, all inequalities should be understood to hold globally unless otherwise indicated, and we omit the arguments of our functions when they are clear.

\section{Main Lyapunov Function CONSTRUCtion}

Fix a continuous function $K: \mathbb{R}^{n} \rightarrow \mathbb{R}^{n \times n}$ that has constants $c, \bar{K}>0$ such that $\xi^{\top} K(\xi) \xi \geq$ $c|\xi|^{2}$ and $|K(\xi)| \leq \bar{K}$ for all $\xi \in \mathbb{R}^{n}$. Let $\hat{\theta}$ denote the state of the estimator of the unknown parameter $\theta \in \mathbb{R}^{p}$ in $\left.\sqrt{3}\right)$, set $(e, \tilde{\theta})=\left(x_{r}-x, \theta-\hat{\theta}\right)$, and choose the augmented dynamics

$$
\dot{x}=\omega(x) \theta+u_{s}(t, x, \hat{\theta}), \quad \dot{\hat{\theta}}=\nu(t, x, \hat{\theta}) .
$$

For simplicity, we choose the adaptive controller

$$
u_{s}(t, x, \hat{\theta})=\dot{x}_{r}(t)-\omega(x) \hat{\theta}+K(e) e, \quad \nu(t, x, \hat{\theta})=-\omega(x)^{\top}\left(x_{r}(t)-x\right)
$$

(see $\mathrm{p}, 10$ below for more general $K, u_{s}$, and $\nu$ ). We have the closed loop error dynamics

$$
\dot{e}=-\omega(x) \tilde{\theta}-K(e) e, \quad \dot{\tilde{\theta}}=\omega(x)^{\top} e,
$$

since $\theta$ is constant. We will take the nonstrict Lyapunov function $V_{1}(e, \tilde{\theta})=\frac{1}{2}|(e, \tilde{\theta})|^{2}$. We also set $V_{4}=V_{2}+V_{3}$, where

$$
V_{2}(t, e, \tilde{\theta})=\tilde{\theta}^{\top} \omega\left(x_{r}(t)\right)^{\top} e \text { and } V_{3}(t, \tilde{\theta})=\frac{1}{T} \tilde{\theta}^{\top}\left[\int_{t-T}^{t} \int_{m}^{t} \omega\left(x_{r}(l)\right)^{\top} \omega\left(x_{r}(l)\right) \mathrm{d} l \mathrm{~d} m\right] \tilde{\theta} .
$$

Recalling the constants from Assumptions 1, 2, we also use the functions

$$
\begin{aligned}
& P_{5}(l)=\frac{2}{c} \int_{0}^{l} P_{4}(m) \mathrm{d} m+\bar{\omega}(B) l, \quad \text { where } P_{4}(l)=\frac{T}{2 \mu}\left[P_{0}+P_{2}(l)+P_{3}(l)\right]^{2}+P_{1}(l)+\frac{c}{2}, \\
& P_{3}(l)=T \bar{\omega}(\sqrt{2 l}+B) \bar{\omega}^{2}(B), \quad P_{2}(l)=\sqrt{2 \ln p} \sup _{i, j}\left\{\left|\frac{\partial \omega_{i j}(q)}{\partial q}\right|:|q| \leq \sqrt{2 l}+B\right\} \bar{\omega}(B), \\
& P_{1}(l)=\bar{\omega}(B) \bar{\omega}(\sqrt{2 l}+B), \quad \text { and } \quad P_{0}=\max \left\{2 \bar{\omega}_{1}(B), 2 \bar{K} \bar{\omega}(B)\right\} .
\end{aligned}
$$


Note that $P_{5} \in C^{1}$ on $[0, \infty)$, and that $|\omega(x)| \leq \bar{\omega}(|e|+B)$ and $\left|\omega\left(x_{r}(t)\right)\right||\omega(x)| \leq P_{1}\left(V_{1}(e, \tilde{\theta})\right)$ for all $t \in \mathbb{R}, x \in \mathbb{R}^{n}$ and $\tilde{\theta} \in \mathbb{R}^{p}$ when $e=x_{r}(t)-x$. Also, the constant $B$ depends only on $x_{r}$, and $\bar{\omega}$ and $\bar{\omega}_{1}$ depend only on $\omega$ and $x_{r}$, so the following construction is a global one:

Theorem 1: Under the preceding assumptions, we can transform the nonstrict Lyapunov function $V_{1}$ into the explicit, global, strict Lyapunov function

$$
V_{5}(t, e, \tilde{\theta})=V_{4}(t, e, \tilde{\theta})+P_{5}\left(V_{1}(e, \tilde{\theta})\right)
$$

for (6) which is therefore UGAS; i.e., there exist functions $\alpha_{1}, \alpha_{2} \in \mathcal{K}_{\infty}$ and a positive definite function $W$ so that the function $V_{5}:[0, \infty) \times \mathbb{R}^{2} \rightarrow[0, \infty)$ defined by (9) satisfies $\alpha_{1}(|(e, \tilde{\theta})|) \leq V_{5}(t, e, \tilde{\theta}) \leq \alpha_{2}(|(e, \tilde{\theta})|)$ for all $(t, e, \tilde{\theta})$ and $\dot{V}_{5}(t, e, \tilde{\theta}) \leq-W(e, \tilde{\theta})$ along all trajectories of the closed loop system, and $\left(x_{r}(t), \theta\right)$ is a UGAS trajectory for (4)-(5). Also, (6) is locally exponentially stable to 0 .

The proof is constructive, leading to explicit formulas for the $\alpha_{i}$ 's and $W$. Notice that the $C^{1}$ property of $V_{5}$ is clear from the regularity of the formulas for the $P_{i}$ 's in (8).

\section{Proof of Theorem 1}

Since $\left|V_{2}(t, e, \tilde{\theta})\right| \leq \bar{\omega}(B)|\tilde{\theta}||e| \leq \bar{\omega}(B) V_{1}$ and $P_{4}(l) \geq c / 2$ everywhere, and since $P_{4}$ is nondecreasing, our formula (9) gives

$$
\begin{aligned}
V_{5}(t, e, \tilde{\theta}) & \geq V_{2}+\frac{2}{c} \int_{0}^{V_{1}} P_{4}(l) \mathrm{d} l+\bar{\omega}(B) V_{1} \geq \frac{1}{2}|e|^{2}+\frac{1}{2}|\tilde{\theta}|^{2}=: \quad \alpha_{1}(|(e, \tilde{\theta})|) \\
V_{5}(t, e, \tilde{\theta}) & \leq \bar{\omega}(B)|\tilde{\theta}||e|+\frac{T}{2}|\tilde{\theta}|^{2} \bar{\omega}^{2}(B)+\frac{2}{c} \int_{0}^{V_{1}} P_{4}(m) \mathrm{d} m+\bar{\omega}(B) V_{1} \\
& \leq \bar{\omega}(B)|\tilde{\theta}||e|+\frac{T}{2}|\tilde{\theta}|^{2} \bar{\omega}^{2}(B)+\frac{1}{2}\left[\frac{2}{c} P_{4}\left(V_{1}\right)+\bar{\omega}(B)\right]\left(|e|^{2}+|\tilde{\theta}|^{2}\right) \\
& \leq\left[\bar{\omega}(B)(1+\bar{\omega}(B) T)+\frac{2}{c} P_{4}\left(|e|^{2}+|\tilde{\theta}|^{2}\right)\right]\left(|e|^{2}+|\tilde{\theta}|^{2}\right)=: \alpha_{2}(|(e, \tilde{\theta})|)
\end{aligned}
$$

everywhere (where the lower bound follows because $V_{3}$ is everywhere nonnegative using the formula for $P_{5}$ in $(8)$, and the upper bound used the relations

$$
\left|\int_{t-T}^{t} \int_{m}^{t} \omega\left(x_{r}(l)\right)^{\top} \omega\left(x_{r}(l)\right) \mathrm{d} l \mathrm{~d} m\right| \leq \frac{T^{2}}{2} \bar{\omega}^{2}(B),
$$

$\int_{0}^{V_{1}} P_{4}(m) \mathrm{d} m \leq P_{4}\left(V_{1}\right) V_{1}$, and the triangular inequality $\left.|e||\tilde{\theta}| \leq \frac{1}{2}|e|^{2}+\frac{1}{2}|\tilde{\theta}|^{2}\right)$ so $V_{5}$ is uniformly proper and positive definite, since $\alpha_{1}, \alpha_{2} \in \mathcal{K}_{\infty}$. Our assumptions on $K$ and (6) readily give

$$
\begin{aligned}
& \dot{V}_{1}=-e^{\top} K(e) e \leq-c|e|^{2} \text { and } \\
& \left.\dot{V}_{2}=\tilde{\theta}^{\top} \omega\left(x_{r}(t)\right)^{\top}[-\omega(x) \tilde{\theta}-K(e) e]+\tilde{\theta}^{\top} \frac{d\left[\omega\left(x_{r}(t)\right)\right]}{d t}\right]^{\top} e+e^{\top} \omega(x) \omega\left(x_{r}(t)\right)^{\top} e .
\end{aligned}
$$

Here and in the sequel, dots indicate time derivatives along the trajectories of (6). By Assumption 1. we have the global inequality $e^{\top} \omega(x) \omega\left(x_{r}(t)\right)^{\top} e \leq P_{1}\left(V_{1}\right)|e|^{2}$, because

$$
|\omega(x)| \leq \bar{\omega}(|e|+B) \leq \bar{\omega}\left(\sqrt{2 V_{1}}+B\right)
$$


everywhere. Also,

$$
\max \left\{-\tilde{\theta}^{\top} \omega\left(x_{r}(t)\right)^{\top} K(e) e, \tilde{\theta}^{\top} \frac{d\left[\omega\left(x_{r}(t)\right)\right]^{\top}}{d t} e\right\} \leq \frac{1}{2} P_{0}|\tilde{\theta} \| e|,
$$

and $\left|\omega(x)-\omega\left(x_{r}(t)\right)\right| \leq|e| \sqrt{n p} \max _{i, j}\left\{\left|\left(\partial \omega_{i j} / \partial x\right)(p)\right|:|p| \leq \sqrt{2 V_{1}}+B\right\}$ gives the estimate

$$
-\tilde{\theta}^{\top} \omega\left(x_{r}(t)\right)^{\top}\left[\omega(x)-\omega\left(x_{r}(t)\right)\right] \tilde{\theta} \leq P_{2}\left(V_{1}\right)|\tilde{\theta}||e| .
$$

(We used $|A| \leq \sqrt{n p} \max _{i, j}\left|a_{i j}\right|$ for any $A=\left[a_{i j}\right] \in \mathbb{R}^{n \times p}$, plus the Mean Value Theorem applied to the entries $\omega_{i j}$ of $\omega$.) Therefore, (12) gives

$$
\begin{aligned}
\dot{V}_{2} & \leq-\tilde{\theta}^{\top} \omega\left(x_{r}(t)\right)^{\top} \omega(x) \tilde{\theta}+P_{0}|\tilde{\theta}||e|+P_{1}\left(V_{1}\right)|e|^{2} \\
& \leq-\tilde{\theta}^{\top} \omega\left(x_{r}(t)\right)^{\top} \omega\left(x_{r}(t)\right) \tilde{\theta}+\left[P_{0}+P_{2}\left(V_{1}\right)\right]|\tilde{\theta}||e|+P_{1}\left(V_{1}\right)|e|^{2}
\end{aligned}
$$

(where the last inequality is by writing $\omega(x)=\omega\left(x_{r}(t)\right)+\left[\omega(x)-\omega\left(x_{r}(t)\right)\right]$ and using (15)). By (6) and our key assumption of the classical PE condition in Assumption 2, we get

$$
\begin{aligned}
\dot{V}_{3}= & \frac{2}{T} \tilde{\theta}^{\top}\left[\int_{t-T}^{t} \int_{m}^{t} \omega\left(x_{r}(l)\right)^{\top} \omega\left(x_{r}(l)\right) \mathrm{d} l \mathrm{~d} m\right] \omega(x)^{\top} e+\tilde{\theta}^{\top} \omega\left(x_{r}(t)\right)^{\top} \omega\left(x_{r}(t)\right) \tilde{\theta} \\
& -\frac{1}{T} \tilde{\theta}^{\top}\left[\int_{t-T}^{t} \omega\left(x_{r}(l)\right)^{\top} \omega\left(x_{r}(l)\right) \mathrm{d} l\right] \tilde{\theta} \\
\leq & \frac{2}{T} \tilde{\theta}^{\top}\left[\int_{t-T}^{t} \int_{m}^{t} \omega\left(x_{r}(l)\right)^{\top} \omega\left(x_{r}(l)\right) \mathrm{d} l \mathrm{~d} m\right] \omega(x)^{\top} e+\tilde{\theta}^{\top} \omega\left(x_{r}(t)\right)^{\top} \omega\left(x_{r}(t)\right) \tilde{\theta}-\frac{\mu}{T}|\tilde{\theta}|^{2} .
\end{aligned}
$$

By Assumption 1 and the relations (11) and (13) above, the function $P_{3}$ from (8) is such that

$$
\frac{2}{T} \tilde{\theta}^{\top}\left[\int_{t-T}^{t} \int_{m}^{t} \omega\left(x_{r}(l)\right)^{\top} \omega\left(x_{r}(l)\right) \mathrm{d} l \mathrm{~d} m\right] \omega(x)^{\top} e \leq P_{3}\left(V_{1}\right)|\tilde{\theta}||e| .
$$

Combining the preceding inequalities and canceling terms, we obtain

$$
\begin{aligned}
\dot{V}_{4}=\dot{V}_{2}+\dot{V}_{3} \leq & -\tilde{\theta}^{\top} \omega\left(x_{r}(t)\right)^{\top} \omega\left(x_{r}(t)\right) \tilde{\theta}+\left[P_{0}+P_{2}\left(V_{1}\right)\right]|\tilde{\theta}||e|+P_{1}\left(V_{1}\right)|e|^{2} \\
& +P_{3}\left(V_{1}\right)|\tilde{\theta}||e|+\tilde{\theta}^{\top} \omega\left(x_{r}(t)\right)^{\top} \omega\left(x_{r}(t)\right) \tilde{\theta}-\frac{\mu}{T}|\tilde{\theta}|^{2} \\
= & \left\{\left[P_{0}+P_{2}\left(V_{1}\right)+P_{3}\left(V_{1}\right)\right]|e|\right\}|\tilde{\theta}|+P_{1}\left(V_{1}\right)|e|^{2}-\frac{\mu}{T}|\tilde{\theta}|^{2} .
\end{aligned}
$$

Applying the inequality $a|\tilde{\theta}| \leq \frac{T}{2 \mu} a^{2}+\frac{\mu}{2 T}|\tilde{\theta}|^{2}$ where $a$ is the term in braces in $\sqrt{19}$ gives

$$
\begin{aligned}
\dot{V}_{4} & \leq \frac{T}{2 \mu}\left[P_{0}+P_{2}\left(V_{1}\right)+P_{3}\left(V_{1}\right)\right]^{2}|e|^{2}+\frac{\mu}{2 T}|\tilde{\theta}|^{2}+P_{1}\left(V_{1}\right)|e|^{2}-\frac{\mu}{T}|\tilde{\theta}|^{2} \\
& \leq P_{4}\left(V_{1}\right)|e|^{2}-\frac{\mu}{2 T}|\tilde{\theta}|^{2} .
\end{aligned}
$$

Since $\dot{V}_{1} \leq-c|e|^{2}$ everywhere, 20 and our choice of $P_{4}$ in $(8)$ give

$$
\begin{aligned}
\dot{V}_{5} & =\dot{V}_{4}+\left[\frac{2}{c} P_{4}\left(V_{1}\right)+\bar{\omega}(B)\right] \dot{V}_{1} \leq \dot{V}_{4}+\frac{2}{c} P_{4}\left(V_{1}\right) \dot{V}_{1} \\
& \leq \dot{V}_{4}-2 P_{4}\left(V_{1}\right)|e|^{2} \leq-P_{4}\left(V_{1}(e, \tilde{\theta})\right)|e|^{2}-\frac{\mu}{2 T}|\tilde{\theta}|^{2},
\end{aligned}
$$

where the first inequality holds because $\bar{\omega}(B) \dot{V}_{1} \leq 0$. By $(10), V_{5}$ is uniformly proper and positive definite. Since $P_{4}(l) \geq c / 2$ for all $l$, we conclude that $V_{5}$ is a strict Lyapunov function for (6). The local exponential stability follows from the positive definite quadratic lower and upper bounds for 
$V_{5}$ and $P_{4}\left(V_{1}(e, \tilde{\theta})\right)|e|^{2}+\frac{\mu}{2 T}|\tilde{\theta}|^{2}$ near 0 ; see 10 . The result readily follows.

\section{Robustness Result}

We illustrate the usefulness of Lyapunov functions for quantifying the effects of uncertainty by showing that when $\omega$ has affine growth, the perturbed error dynamics

$$
\dot{e}=-\omega(x)[\tilde{\theta}+\delta(t)]-K(e) e, \quad \dot{\tilde{\theta}}=\omega(x)^{\top} e,
$$

obtained by replacing $\theta$ with $\theta+\delta(t)$ in $(3)$ (i.e., the uncertain plant is $\dot{x}=\omega(x)(\theta+\delta(t))+u_{s}$ ) and using the controller (5), is ISS with respect to suitably bounded uncertainties $\delta(t)$. We always assume that $\delta(t)$ is bounded in the essential supremum norm by a constant $\bar{\delta}>0$ that we specify shortly, and that Assumptions 1,2 hold for some positive constants $B, \mu$ and $T$. We take $K(e) \equiv$ $c I_{n}$, where the constant $c \geq 1$ will depend on the choice of $\bar{\delta}$; see 28) for the condition on $c$ for a given bound $\bar{\delta}$. Finally, we assume that there are constants $\omega_{M} \geq \max \{1, \bar{\omega}(B)\}$ and $\eta>0$ such that the following affine growth condition holds:

$$
|\omega(x)| \leq \omega_{M}+\eta|x| \text { and }\left|\frac{\partial \omega_{i j}}{\partial x}(x)\right| \leq \omega_{M} \forall x, i, j .
$$

Hence $\bar{\omega}_{1}(B) \leq \omega_{M} \sqrt{n p} B$. We prove that 22 is ISS when $c$ is big enough by explicitly constructing an ISS Lyapunov function. We use the constants

$$
\begin{aligned}
& \Delta_{1}=\frac{T}{\mu} \omega_{M}^{6}[2 \max \{c,(1+B) \sqrt{n p}\}+T\{1+\eta(B+2)\}]^{2}+\eta \omega_{M}(B+2)+\omega_{M}^{2}+\frac{c}{2}, \\
& C_{1}=\frac{2 T}{\mu} \omega_{M}^{4}[\sqrt{n p}+\eta T]^{2}+2 \eta \omega_{M}+0.5 \sqrt{c}, \text { and } \Delta_{2}=\omega_{M}+\frac{2 \Delta_{1}}{c} .
\end{aligned}
$$

Note that $\Delta_{1} \geq C_{1}$, since $c \geq 1$ and $\omega_{M} \geq 1$. Also, (23) is not required in Theorem 11, so the following construction cannot be used to prove Theorem 1 by setting $\delta \equiv 0$.

Theorem 2: Let the preceding assumptions hold. For each constant $\bar{\delta}>0$, we can compute a constant $c \geq 1$ (depending on $\bar{\delta}$ ) so that 22) with uncertainties $\delta: \mathbb{R} \rightarrow \bar{\delta} \mathcal{B}_{p}$ bounded by $\bar{\delta}$ admits the ISS Lyapunov function

$$
\begin{aligned}
V_{5}(t, e, \tilde{\theta})= & \tilde{\theta}^{\top} \omega\left(x_{r}(t)\right)^{\top} e+\frac{1}{T} \tilde{\theta}^{\top}\left[\int_{t-T}^{t} \int_{m}^{t} \omega\left(x_{r}(l)\right)^{\top} \omega\left(x_{r}(l)\right) \mathrm{d} l \mathrm{~d} m\right] \tilde{\theta} \\
& +\Delta_{2} V_{1}(e, \tilde{\theta})+\frac{C_{1}}{c} V_{1}^{2}(e, \tilde{\theta})
\end{aligned}
$$

and so is ISS with respect to the uncertainty $\delta: \mathbb{R} \rightarrow \bar{\delta} \mathcal{B}_{p}$.

Proof: We use the notation from Theorem 1. We may assume that $\omega_{M}=\bar{\omega}(B) \geq 1$. Then

$$
\begin{aligned}
& P_{0}(l) \leq 2 \omega_{M} \max \{c, \sqrt{n p} B\}, \quad P_{1}(l) \leq \omega_{M}^{2}+\eta \omega_{M}(\sqrt{2 l}+B), \quad P_{2}(l) \leq \omega_{M}^{2} \sqrt{2 \ln p}, \\
& P_{3}(l) \leq T \omega_{M} P_{1}(l), \text { and } P_{4}(l)=\frac{T}{2 \mu}\left[P_{0}+P_{2}(l)+P_{3}(l)\right]^{2}+P_{1}(l)+\frac{c}{2} \leq \Delta_{1}+C_{1} l
\end{aligned}
$$


by $(23)$. By enlarging $P_{4}$ as necessary without relabeling, we assume that $P_{4}(l)=\Delta_{1}+C_{1} l$ in the sequel. Therefore, $V_{5}$ from Theorem 1 takes the form (25). One easily checks that Theorem 1 remains true when $P_{4}$ is enlarged in this way. Notice that Assumption 1 and 23 give $|\omega(x)| \leq$ $\omega_{M}+\eta B+\eta|e|$ everywhere. Therefore, using 21 and 22, , we get:

$$
\begin{gathered}
\dot{V}_{5} \leq-\left[\Delta_{1}+C_{1} V_{1}(e, \tilde{\theta})\right]|e|^{2}-\frac{\mu}{2 T}|\tilde{\theta}|^{2}+\left(\omega_{M}+\eta B+\eta|e|\right)\left|\frac{\partial V_{5}}{\partial e}(t, e, \tilde{\theta})\right||\delta(t)| \\
\text { and }\left|\frac{\partial V_{5}}{\partial e}(t, e, \tilde{\theta})\right| \\
\leq\left|\omega\left(x_{r}(t)\right) \tilde{\theta}\right|+\left[\Delta_{2}+2 \frac{C_{1}}{c} V_{1}(e, \tilde{\theta})\right]|e| \\
\leq \omega_{M}|\tilde{\theta}|+\left[\Delta_{2}+2 \frac{C_{1}}{c} V_{1}(e, \tilde{\theta})\right]|e| .
\end{gathered}
$$

Assume that

$$
\bar{\delta} \leq \min \left\{\frac{C_{1}}{8 \omega_{M} \eta}, \frac{c}{8\left(\omega_{M}+\eta[1+B]\right)}, \frac{c}{C_{1}\left(\omega_{M}+\eta[1+B]\right)} \min \left\{0.9 \Delta_{1}, \frac{\mu}{3 T}\right\}\right\} .
$$

We consider two cases. Case 1: $|e| \geq 1$. In this case, dropping $-\Delta_{1}|e|^{2}-\frac{\mu}{2 T}|\tilde{\theta}|^{2}$ in $[27$ gives

$$
\begin{aligned}
\dot{V}_{5} \leq & -\frac{C_{1}}{4}\left(|e|^{2}+|\tilde{\theta}|^{2}\right)-\frac{C_{1}}{3} V_{1}(e, \tilde{\theta})|e|^{2}-\frac{C_{1}}{12}|e|^{4}+\omega_{M}\left(\omega_{M}+\eta B\right)|\tilde{\theta}||\delta(t)| \\
& +\omega_{M} \eta|e||\tilde{\theta}||\delta(t)|+\left\{|e|^{2}\right\}\left\{\left[\omega_{M}+\eta(1+B)\right] \Delta_{2}|\delta(t)|\right\} \\
& +2 \frac{C_{1}}{c}\left(\omega_{M}+\eta[B+1]\right) V_{1}|e|^{2}|\delta(t)| .
\end{aligned}
$$

Applying the relations

$$
|\tilde{\theta}||\delta(t)| \leq \frac{C_{1}|\tilde{\theta}|^{2}}{10 \omega_{M}\left(\omega_{M}+\eta B\right)}+\frac{5}{2 C_{1}} \omega_{M}\left(\omega_{M}+\eta B\right)|\delta(t)|^{2} \text { and }|\tilde{\theta}||e| \leq \frac{1}{2}|\tilde{\theta}|^{2}+\frac{1}{2}|e|^{2}
$$

to the fourth and fifth terms on the right side of 29p, using the relation $a b \leq C_{1} a^{2} / 12+3 b^{2} / C_{1}$ with $a=|e|^{2}$ on the terms in braces in (29), and recalling our assumption (28) on $\bar{\delta}$ gives

$$
\dot{V}_{5} \leq-\frac{C_{1}}{40}|(e, \tilde{\theta})|^{2}+\frac{5}{2 C_{1}} \omega_{M}^{2}\left(\omega_{M}+\eta B\right)^{2}|\delta(t)|^{2}+\frac{3}{C_{1}}\left[\left(\omega_{M}+\eta B\right) \Delta_{2}+\eta \Delta_{2}\right]^{2}|\delta(t)|^{2} .
$$

Case 2: $|e| \leq 1$. In this case, (27) gives

$$
\begin{aligned}
\dot{V}_{5} \leq & -\left[\Delta_{1}+C_{1} V_{1}(e, \tilde{\theta})\right]|e|^{2}-\frac{\mu}{2 T}|\tilde{\theta}|^{2}+\omega_{M}\left(\omega_{M}+\eta[B+1]\right)|\tilde{\theta}||\delta(t)| \\
& +\left(\omega_{M}+\eta[B+1]\right)\left[\Delta_{2}+\frac{C_{1}}{c}\left(|e|^{2}+|\tilde{\theta}|^{2}\right)\right]|\delta(t)| \\
\leq & -\frac{\Delta_{1}}{10}|e|^{2}-\frac{\mu}{15 T}|\tilde{\theta}|^{2}+\frac{5 T\left\{\omega_{M}\left(\omega_{M}+\eta[B+1]\right)\right\}^{2}}{2 \mu}|\delta(t)|^{2}+\Delta_{2}\left[\omega_{M}+\eta(B+1)\right]|\delta(t)|,
\end{aligned}
$$

where the last inequality followed from dropping the term $-C_{1} V_{1}(e, \tilde{\theta})|e|^{2}, 288$, and

$$
|\tilde{\theta}||\delta(t)| \leq \frac{\mu}{10 T \omega_{M}\left(\omega_{M}+\eta[B+1]\right)}|\tilde{\theta}|^{2}+\frac{10 T \omega_{M}\left(\omega_{M}+\eta[B+1]\right)}{4 \mu}|\delta(t)|^{2} .
$$

Conditions (31) and (32) and the uniform proper and positive definiteness of $V_{5}$ (noted in (10)) imply that $V_{5}$ is an ISS Lyapunov function for 22 when $|\delta|_{\infty} \leq \bar{\delta}$. The theorem follows because (a) the existence of an ISS Lyapunov function implies the ISS property (cf. [18] or Remark 1 below) and (b) the right hand side of (28) can be made as large as desired by choosing a big enough constant $c$ depending on $\bar{\delta}$. 
Remark 1: The explicit ISS Lypanov function (25) for (22) leads to explicit expressions for $\beta$ and $\gamma$ in the ISS estimate for [22), as follows. Define $\alpha_{1}, \alpha_{2}, \alpha_{3}, \alpha_{4}, \alpha \in \mathcal{K}_{\infty}$ by (10),

$$
\begin{aligned}
\alpha_{3}(r)= & \min \left\{\frac{C_{1}}{40}, \frac{\mu}{15 T}\right\} r^{2}, \alpha(r)=\min \left\{r, \alpha_{3} \circ \alpha_{2}^{-1}(r)\right\}, \text { and } \\
\alpha_{4}(r)= & \left\{\frac{5}{2 C_{1}} \omega_{M}^{2}\left(\omega_{M}+\eta B\right)^{2}+\frac{3}{C_{1}}\left[\left(\omega_{M}+\eta B\right) \Delta_{2}+\eta \Delta_{2}\right]^{2}+\frac{5 T \omega_{M}^{2}\left(\omega_{M}+\eta[B+1]\right)^{2}}{2 \mu}\right\} r^{2} \\
& +\Delta_{2}\left[\omega_{M}+\eta(B+1)\right] r .
\end{aligned}
$$

Then $\alpha_{1}(|(e, \tilde{\theta})|) \leq V_{5}(t, e, \tilde{\theta}) \leq \alpha_{2}(|(e, \tilde{\theta})|)$ and $\dot{V}_{5} \leq-\alpha\left(V_{5}\right)+\alpha_{4}\left(|\delta|_{\infty}\right)$ along all trajectories of 222) when $\bar{\delta}$ satisfies (28) (by (10) and (31)-(32)), and then the explicit formulas for $\beta$ and $\gamma$ in the ISS estimate follow by standard arguments [17], [18].

\section{ApPLiCATION: RÖSSLER SySTEM}

We illustrate our Lyapunov function constructions using the controlled Rössler dynamics

$$
\dot{x}_{1}=a x_{1}+x_{2}+w_{1}, \quad \dot{x}_{2}=-x_{1}-x_{3}+w_{2}, \quad \dot{x}_{3}=b+x_{3}\left[x_{2}-c\right]+w_{3}
$$

with unknown parameters $a, b$, and $c$ and control vector $w=\left(w_{1}, w_{2}, w_{3}\right)$. The Rössler model (for the case of no controls) was introduced in [14] and has been extensively studied in the context of chaotic attractors [8]. The system [34 can be written in the form $\dot{x}=\omega(x) \theta+u$ by taking the change of feedback

$$
u=w-\left[\begin{array}{ccc}
0 & -1 & 0 \\
1 & 0 & 1 \\
0 & -x_{3} & 0
\end{array}\right] x, \quad \omega(x)=\left[\begin{array}{ccc}
x_{1} & 0 & 0 \\
0 & 0 & 0 \\
0 & 1 & -x_{3}
\end{array}\right], \quad \text { and } \quad \theta=\left[\begin{array}{c}
a \\
b \\
c
\end{array}\right] .
$$

Let us show that the PE condition from Assumption 2 is satisfied for an appropriate class of reference trajectories. Fix any $C^{1}$ reference trajectory of the form $x_{r}(t)=\left(x_{1 r}(t), x_{2 r}(t), \cos (t)\right)$ that satisfies our Assumption 1 for some constant $B>1$ and that admits a constant $\mu_{o} \in(0,1)$ such that $\int_{t-2 \pi}^{t} x_{1 r}^{2}(l) \mathrm{d} l \geq \mu_{o}$ for all $t \in \mathbb{R}$. Then

$$
\int_{t-2 \pi}^{t} \omega\left(x_{r}(l)\right)^{\top} \omega\left(x_{r}(l)\right) \mathrm{d} l=\left[\begin{array}{ccc}
\int_{t-2 \pi}^{t} x_{1 r}^{2}(l) \mathrm{d} l & 0 & 0 \\
0 & 2 \pi & 0 \\
0 & 0 & \pi
\end{array}\right],
$$

so Assumption 2 and our growth assumption (23) hold with $\mu=\mu_{o}, T=2 \pi, \eta=2$, and $\omega_{M}=2(B+1)$. Hence, for any constant $c \geq 1$, the error dynamics for the Rössler system (34) with the adaptive controller (5) and $K(e) \equiv c I_{n}$ admits a global strict Lyapunov function of the form (25) and so is UGAS. Also, Theorem 2 shows that (25) is an ISS Lyapunov function when $\theta$ is perturbed by time varying additive uncertainty $\delta$, where $c$ depends on the choice of $\bar{\delta}$. 


\section{EXtension: More General FeEdBACKS}

Theorem 1 assumes that the systems are adaptively controlled by (5), and that the known nonstrict Lyapunov function $V_{1}$ is $\frac{1}{2}|(e, \tilde{\theta})|^{2}$. Let us show how these assumptions can be relaxed.

We first assume that Assumptions 1 , 2 hold, and that there exist a $C^{1}$ function $\nu(t, e, \hat{\theta})$, a bounded $C^{1}$ function $K(e)$, a uniformly proper and positive definite $C^{1}$ function $V_{1}$, a positive definite function $W_{1}$, a continuous positive increasing function $P_{\nu}$, and a constant $c>0$ such that (i) $|\nu(t, e, \hat{\theta})| \leq P_{\nu}\left(V_{1}\right)|e|$ everywhere, (ii) $\dot{V}_{1}(t, e, \tilde{\theta}) \leq-W_{1}(e)$ along all trajectories of

$$
\dot{e}=-\omega(x) \tilde{\theta}-K(e) e, \quad \dot{\tilde{\theta}}=-\nu(t, e, \hat{\theta}),
$$

and (iii) $W_{1}(e) \geq c|e|^{2}$ everywhere, where $e$ and $\tilde{\theta}$ are as defined in Section III. In other words, we replace $\nu=-\omega(x)^{\top} e$ from Theorem 1 with a general adaptation law that could include, for example, projection operators, least-squares estimators, and prediction-error-based estimators [3], [16]. A slight variant of the proof of Theorem 1 constructs a function $P_{5}$ so that (9) is a global strict Lyapunov function for (35) when (i)-(iii) are satisfied.

A different generalization is as follows. Let $e$ and $\tilde{\theta}$ be as in Section III, and let $\omega$ and $x_{r}$ satisfy Assumptions 1,2. Assume that there exist a (possibly unbounded) matrix function $K$ with $C^{1}$ entries, a $C^{1}$ uniformly proper and positive definite function $V_{a}(t, z)$, a positive definite function $W_{a}(z)$, and a continuous positive function $\Delta$ so that $(\mathrm{G} 1) \dot{V}_{a}(t, z) \leq-W_{a}(z)$ along all trajectories of $\dot{z}=-K\left(z+x_{r}(t)\right) z,(\mathrm{G} 2)$ all the second partial derivatives $\partial^{2} V_{a} / \partial z_{i} \partial z_{j}$ are uniformly bounded in $z$, and (G3) $W_{a}(z) \geq \Delta(|z|)|z|^{2}$ everywhere. Taking (4) and

$$
u_{s}=\dot{x}_{r}(t)-\omega(x) \hat{\theta}+K\left(e+x_{r}(t)\right) e, \quad \nu=-\left[\frac{\partial V_{a}}{\partial e}(t, e) \omega(x)\right]^{\top}, \quad V_{1}(t, e, \tilde{\theta})=V_{a}(t, e)+\frac{1}{2}|\tilde{\theta}|^{2}
$$

guarantees that the time derivative of $V_{1}$ along the trajectories of the closed loop error dynamics

$$
\dot{e}=-\omega(x) \tilde{\theta}-K\left(e+x_{r}(t)\right) e, \quad \dot{\tilde{\theta}}=\left[\frac{\partial V_{a}}{\partial e}(t, e) \omega(x)\right]^{\top}
$$

satisfies $\dot{V}_{1}(t, e, \tilde{\theta}) \leq-W_{a}(e)$. Setting $V_{4}=V_{2}+\frac{1}{2} V_{3}$ with $V_{2}$ and $V_{3}$ from 77 , we prove:

Theorem 3: Let Assumptions 1, 2 and G1-G3 hold. Then we can explicitly construct a function $\kappa_{4} \in \mathcal{K}_{\infty} \cap C^{1}$ so that $V_{5}(t, e, \tilde{\theta})=\kappa_{4}\left(V_{1}(t, e, \tilde{\theta})\right)+V_{4}(t, e, \tilde{\theta})$ is a global strict Lyapunov function for the error dynamics 36 which are therefore UGAS.

Sketch of Proof. The proof is similar to the proof of Theorem 1 so we only provide a sketch. The fact that $\dot{e}=-\omega\left(x_{r}\right) \tilde{\theta}-K\left(e+x_{r}\right) e-\left[\omega(x)-\omega\left(x_{r}\right)\right] \tilde{\theta}$ easily gives

$$
\begin{aligned}
\dot{V}_{2}= & -\tilde{\theta}^{\top} \omega\left(x_{r}(t)\right)^{\top} \omega\left(x_{r}(t)\right) \tilde{\theta}+\left[\frac{\partial V_{a}}{\partial e}(t, e) \omega(x)\right] \omega\left(x_{r}(t)\right)^{\top} e+\tilde{\theta}^{\top} \frac{d \omega\left(x_{r}(t)\right)^{\top}}{d t} e \\
& -\tilde{\theta}^{\top} \omega\left(x_{r}(t)\right)^{\top} K\left(e+x_{r}\right) e-\tilde{\theta}^{\top} \omega\left(x_{r}(t)\right)^{\top}\left[\omega(x)-\omega\left(x_{r}(t)\right)\right] \tilde{\theta} .
\end{aligned}
$$


Applying the Mean Value Theorem to $e \mapsto \frac{\partial V_{a}}{\partial e}(t, e) \omega\left(e+x_{r}(t)\right)$ and $\omega$, one can find a positive increasing function $\kappa_{1}$ such that

$$
\begin{aligned}
& {\left[\frac{\partial V_{a}}{\partial e}(t, e) \omega\left(e+x_{r}(t)\right)\right] \omega\left(x_{r}(t)\right)^{\top} e \leq \kappa_{1}(|e|)|e|^{2}} \\
& \quad-\tilde{\theta}^{\top} \omega\left(x_{r}(t)\right)^{\top} K\left(e+x_{r}\right) e \leq \kappa_{1}(|e|)|\tilde{\theta}||e|
\end{aligned}
$$

and $-\tilde{\theta}^{\top} \omega\left(x_{r}(t)\right)^{\top}\left[\omega(x)-\omega\left(x_{r}(t)\right)\right] \tilde{\theta} \leq\left|\omega\left(x_{r}(t)\right) \tilde{\theta}\right| \kappa_{1}(|e|)|\tilde{\theta}||e|$. We deduce from 37 that

$$
\begin{aligned}
\dot{V}_{2} \leq & -\tilde{\theta}^{\top} \omega\left(x_{r}(t)\right)^{\top} \omega\left(x_{r}(t)\right) \tilde{\theta}+\kappa_{1}(|e|)|e|^{2}+\left[\bar{\omega}_{1}+\kappa_{1}(|e|)\right]|\tilde{\theta}||e| \\
& +\left\{\left|\omega\left(x_{r}(t)\right) \tilde{\theta}\right|\right\}\left\{\kappa_{1}(|e|)|\tilde{\theta}||e|\right\} \\
\leq & -\frac{1}{2} \tilde{\theta}^{\top} \omega\left(x_{r}(t)\right)^{\top} \omega\left(x_{r}(t)\right) \tilde{\theta}+\kappa_{1}(|e|)|e|^{2}+\left[\bar{\omega}_{1}+\kappa_{1}(|e|)\right]|\tilde{\theta}||e|+\frac{1}{2} \kappa_{1}^{2}(|e|)|\tilde{\theta}|^{2}|e|^{2},
\end{aligned}
$$

by applying the relation $a b \leq \frac{1}{2} a^{2}+\frac{1}{2} b^{2}$ to the terms in braces. Again applying the Mean Value Theorem, we can readily construct an increasing positive function $\kappa_{2}$ such that

$$
\begin{aligned}
\dot{V}_{3} & \leq \tilde{\theta}^{\top} \omega\left(x_{r}(t)\right)^{\top} \omega\left(x_{r}(t)\right) \tilde{\theta}-\frac{1}{T} \tilde{\theta}^{\top}\left[\int_{t-T}^{t} \omega\left(x_{r}(s)\right)^{\top} \omega\left(x_{r}(s)\right) d s\right] \tilde{\theta}+\kappa_{2}(|e|)|\tilde{\theta}||e| \\
& \leq \tilde{\theta}^{\top} \omega\left(x_{r}(t)\right)^{\top} \omega\left(x_{r}(t)\right) \tilde{\theta}-\frac{\mu}{T}|\tilde{\theta}|^{2}+2 \kappa_{2}(|e|)|\tilde{\theta}||e|,
\end{aligned}
$$

where the second inequality is by our key PE assumption. Hence, by applying the relation $a b \leq$ $\frac{\mu}{4 T} a^{2}+\frac{T}{\mu} b^{2}$ with $a=|\tilde{\theta}|$, we deduce from $\sqrt{39}$ and 40 that $V_{4}=V_{2}+\frac{1}{2} V_{3}$ satisfies

$$
\begin{aligned}
\dot{V}_{4} & \leq-\frac{\mu}{2 T}|\tilde{\theta}|^{2}+\kappa_{1}(|e|)|e|^{2}+\{|\tilde{\theta}|\}\left\{\left[\bar{\omega}_{1}+\kappa_{1}(|e|)+\kappa_{2}(|e|)+\frac{1}{2} \kappa_{1}^{2}(|e|)|e||\tilde{\theta}|\right]|e|\right\} \\
& \leq-\frac{\mu}{4 T}|\tilde{\theta}|^{2}+\kappa_{1}(|e|)|e|^{2}+\frac{T}{\mu}\left[\bar{\omega}_{1}+\kappa_{1}(|e|)+\kappa_{2}(|e|)+\frac{1}{2} \kappa_{1}^{2}(|e|)|\tilde{\theta}||e|\right]^{2}|e|^{2} .
\end{aligned}
$$

One can construct an increasing positive continuous function $\kappa_{3}$ such that

$$
\kappa_{3}\left(V_{1}(t, e, \tilde{\theta})\right) W_{a}(e) \geq \kappa_{1}(|e|)|e|^{2}+\frac{T}{\mu}\left[\bar{\omega}_{1}+\kappa_{1}(|e|)+\kappa_{2}(|e|)+\frac{1}{2} \kappa_{1}^{2}(|e|)|\tilde{\theta}||e|\right]^{2}|e|^{2} .
$$

(For example, first find an increasing positive continuous function $\kappa_{3}^{o}$ so that $\kappa_{3}^{o}\left(V_{1}(t, e, \tilde{\theta})\right)|e|^{2}$ majorizes the right hand side of 42 , then take $\kappa_{3}(r)=\kappa_{3}^{o}(r) / \Delta\left(\underline{\alpha}^{-1}(r)\right)$ where $\underline{\alpha} \in \mathcal{K}_{\infty}$ is chosen so that $V_{1}(t, e, \tilde{\theta}) \geq \underline{\alpha}(|(e, \tilde{\theta})|)$ everywhere and $\Delta$ is assumed without loss of generality to be decreasing.) Consequently,

$$
\dot{V}_{4} \leq-\frac{\mu}{4 T}|\tilde{\theta}|^{2}+\kappa_{3}\left(V_{1}(t, e, \tilde{\theta})\right) W_{a}(e) .
$$

One checks that $z \mapsto \inf _{t} V_{a}(t, z)$ is bounded below by a positive definite quadratic function near 0.1 Hence, we can choose $\kappa_{4} \in \mathcal{K}_{\infty} \cap C^{1}$ so that $\kappa_{4}^{\prime} \geq 2 \kappa_{3}+1$ and so that $V_{5}$ is uniformly proper and positive definite and satisfies $\dot{V}_{5} \leq-\frac{\mu}{4 T}|\tilde{\theta}|^{2}-\kappa_{3}\left(V_{1}\right) W_{a}(e)$ along all trajectories of 36 .

\footnotetext{
${ }^{1}$ To see why, let $\underline{\Delta}>0$ be a lower bound for $\Delta$ on $\mathcal{B}_{n}$. Let $\bar{K}>0$ be a bound for $K$ on $(1+B) \mathcal{B}_{n}$. Reducing $\underline{\Delta}$, we can assume that all trajectories of $\dot{z}=-K\left(z+x_{r}(t)\right) z$ with initial conditions $z\left(t_{o}\right)=z_{o} \in \Delta \mathcal{B}_{n}$ stay in $\mathcal{B}_{n}$. Along any such trajectory, $(d / d t)\left\{V_{a}(t, z)-\underline{\Delta}|z|^{2} /\{4 \bar{K}\}\right\} \leq-\underline{\Delta}|z|^{2}+\underline{\Delta}|z|^{2} / 2 \leq 0$, hence $V_{a}\left(t_{o}, z_{o}\right)-\underline{\Delta}\left|z_{o}\right|^{2} /\{4 \bar{K}\} \geq V_{a}(t, z(t))-\underline{\Delta}|z(t)|^{2} /\{4 \bar{K}\} \rightarrow$ 0 as $t \rightarrow+\infty$. Therefore, $\inf _{t} V_{a}(t, z) \geq \Delta|z|^{2} /\{4 \bar{K}\}$ on $\Delta \mathcal{B}_{n}$.
} 


\section{CONCLUSIONS}

We built explicit global strict Lyapunov functions for general classes of adaptively controlled nonlinear systems. This made it possible to quantify the effects of uncertainty using ISS. It would be useful to extend our work to systems that are not necessarily affine in the parameter vector, or where the current state is unknown; i.e. adaptive output feedback stabilization.

\section{REFERENCES}

[1] C. De Persis, R. De Santis, and A.S. Morse, "Supervisory control with state-dependent dwell-time logic and constraints," Automatica, vol. 40, no. 2, pp. 269-275, 2004.

[2] W. Hahn, The Stability of Motion, Berlin, Germany: Springer-Verlag, 1967.

[3] P. Ioannou and J. Sun, Robust Adaptive Control, Englewood Cliffs, NJ: Prentice Hall, 1996.

[4] A. Isidori, Nonlinear Control Systems, Vol. II, London, UK: Springer, 1999.

[5] M. Krstić, "Invariant manifolds and asymptotic properties of adaptive nonlinear stabilizers," IEEE Trans. Automat. Control, vol. 41, no. 6, pp. 817-829, 1996.

[6] A. Loría, R. Kelly, and A.R. Teel, "Uniform parametric convergence in the adaptive control of mechanical systems," European J. Control, vol. 11, pp. 87-100, 2005.

[7] A. Loria, E. Panteley, D. Popovic, and A. Teel, " $\delta$-persistency of excitation: a necessary and sufficient condition for uniform attractivity," in Proceedings of the 41st IEEE Confence on Decision and Control, pp. 3506-3511, 2002.

[8] A. Loría and A. Zavala-Río, "Adaptive tracking control of chaotic systems with applications to synchronisation," IEEE Trans. Circuits and Systems, vol. 54, issue 9, pp. 2019-2029, 2007.

[9] F. Mazenc and M. Malisoff, "Further constructions of control-Lyapunov functions and stabilizing feedbacks for systems satisfying the Jurdjevic-Quinn conditions,” IEEE Trans. Automat. Control, vol. 51, no. 2, pp. 360-365, 2006.

[10] F. Mazenc and D. Nesic, "Strong Lyapunov functions for systems satisfying the conditions of La Salle," IEEE Trans. Automat. Control, vol. 49, no. 6, pp. 1026-1030, 2004.

[11] F. Mazenc and D. Nesic, "Lyapunov functions for time varying systems satisfying generalized conditions of Matrosov theorem," Mathematics of Control, Signals, and Systems, vol. 19, no. 2, pp. 151-182, 2007.

[12] A.P. Morgan and K.S. Narendra, "On the uniform asymptotic stability of certain linear nonautonomous differential equations," SIAM J. Control Optim., vol. 15, no. 1, pp. 5-25, 1977.

[13] K.S. Narendra and A.M. Annaswamy, "Persistent excitation in adaptive systems," International J. Control, vol. 45, no. 1, pp. 127-160, 1987.

[14] O.E. Rössler, “An equation for continuous chaos,” Physics Letters A, vol. 57, pp. 397-398, 1976.

[15] J.-J. Slotine and W. Li, "Theoretical issues in adaptive manipulator control," in Proceedings of the 5th Yale Workshop on Applied Adaptive Systems Theory, pp. 252-258, 1987.

[16] J.-J. Slotine and W. Li, Applied Nonlinear Control, Englewood Cliffs, NJ: Prentice Hall, 1991.

[17] E.D. Sontag, "Smooth stabilization implies coprime factorization," IEEE Trans. Automat. Control, vol. 34, no. 4, pp. 435-443, 1989.

[18] E.D. Sontag, "Input-to-State Stability: Basic concepts and results," in Nonlinear and Optimal Control Theory, P. Nistri and G. Stefani, Eds., Berlin, Germany: Springer, 2006, pp. 163-220.

[19] E.D. Sontag and A.R. Teel, "Changing supply functions in input/state stable systems," IEEE Trans. Automat. Control, vol. 40, no. 8, pp. 1476-1478, 1995. 